\title{
Multiband Filter Design Using Generalized Mapping Functions and Synthesis with Lumped Resonators
}

\author{
Serkan YILDIZ ${ }^{1}$, Ahmet AKSEN ${ }^{2}$, Sedat KILINC ${ }^{3}$, Siddik B. YARMAN ${ }^{3}$ \\ ${ }^{1}$ The Scientific and Technological Research Council of Turkey, Kocaeli, Turkey \\ ${ }^{2}$ Department of Electrical and Electronics Engineering, Işı1k University, Istanbul, Turkey \\ ${ }^{3}$ Department of Electrical and Electronics Engineering, Istanbul University, Istanbul, Turkey
}

yildiz.serkan@tubitak.gov.tr, ahmet.aksen@isikun.edu.tr, sedat.kilinc@istanbul.edu.tr, yarman@istanbul.edu.tr

Submitted September 29, 2019 / Accepted March 9, 2020

\begin{abstract}
In this paper, a new multiband frequency mapping function is proposed to design multiband filter. The presented mapping function is a generalized form of the sequential low pass to band pass (LPtoBP) transformation. The multiband filter design is based on the application of the frequency mapping function on a LP prototype. The synthesis of the resulting multiband filter is obtained by lumped element resonators. Several examples are presented to validate the proposed design approach. A triple band filter implementation and measurement results are presented.
\end{abstract}

\section{Keywords}

Multiband filter, multiband mapping, frequency transformation, dual band, triple band

\section{Introduction}

Multiband filters have become essential parts for most of RF/MW systems in recent years [1], [2]. A multiband transceiver provides transmission of fundamental frequency and the reception of its second or third harmonic on a single RF path. Therefore, significant size reduction can be achieved compared to using several single band-pass filters in parallel configuration. Besides the robustness of the system, also overall RF performance is enhanced since using multiband filters eliminates the needs for extra RF modules such as switches, combiners, connectors etc.

In general multiband systems are considered as an alternative solution to UWB systems [3]. Through the development of wireless communication technologies such as GSM900, UMTS, 4G, 5G, etc., RF hardware must be designed to provide services to those protocols which have distinct frequency bands. Proper filtering at the RF frontend of wireless devices is essential for most of applications. Ideally the unnecessary frequency bands between adjacent pass bands must be filtered out to avoid the degenerative effects such as interference, noise, harmonics, heat etc. [4]. This can be achieved by employing multiband filters in RF systems.

Several design approaches were published in literature for the synthesis and implementation of multiband filters. The analytical approaches for this problem are focused on two main tracks. The first is to establish the explicit parametric definition of multiband two-port network in terms of zeros and poles of driving point impedance or admittance [5-8]. Even if an explicit mathematical definition of a multiband filter is established, the synthesis of the multiband filter functions is resulted mostly in unrealizable topologies [9], [10].

In the second approach, frequency transformations and network mapping techniques are used. This approach maps the scaled pass-band characteristic of a pre-assigned prototype network into multiband characteristic. The main drawback in this method is the generation of a suitable mapping functions and their application on a prototype network [1]. Several studies are published in this track. A major study [11] employs finite transmission zeros which are generated by suitable dual band frequency transformation at stop band. In [12], a single band to multi-resonance mapping function is presented from a synthesis point of view. The proposed transformation function is a Foster reactance function including series and shunt resonators. The values of the elements are used in transformation functions at selected resonance frequencies. Creating multiple pass-bands with defined bandwidths does not seem to be possible with this technique. In [13], the multiband mapping functions are well elaborated. It has been presented that by using an intermediate unsymmetrical transformation step, it is possible to generate desired number of pass-bands although the intermediate step is not clearly explained. Several other studies are also published in this fashion with different mapping functions which introduce alternative solutions to multiband filters with different topologies [14-17].

From the designer point of view, the most preferred way is, to have a simple parametric form of multiband mapping function in terms of the desired number of passbands and their bandwidths. Moreover, explicit relations 
should be provided for the synthesis of multiband filter from prototype network.

In this paper, a multiband frequency mapping function for the design of multiband filters is proposed. The mapping function is a generalized and improved version of sequential LPtoBP transformation. The presented approach does not require any intermediate step to generate multiband frequency mapping function. It has been shown that the proposed generalized compact mapping function yields the same results as those sequential approaches used in [17] and [18]. The paper provides an explicit proof to validate these relations. Moreover, the proposed mapping function enables the process of generating the odd number of pass-bands, while the classical sequential LPtoBP mapping functions [16-19] do not.

In this technique, first the multiband mapping function is determined according to assigned band specifications. Then any LP prototype network of Chebyshev, Elliptical or Butterworth, type is chosen as reference. As a final step, the synthesis of the multiband filter is performed by the transformation of each element of the LP prototype to its multiband correspondent. As a result, the multiband network is achieved which will be composed of $\mathrm{LC}$ resonators.

In Sec. 2 of this paper, the construction of the multiband frequency transformation through the sequential LPtoBP transformation is introduced. In Sec. 3, the lumped synthesis of the transformed reactance functions and their related circuit topologies are presented. In Sec. 4, the dual band filter application in [18] is re-visited with the proposed technique to demonstrate the relation between the proposed technique and the sequential LPtoBP transformation method. In Sec. 5, a triple band filter design, application, realization steps and measurement results are presented.

\section{The Multiband Mapping Function}

The aim of most of the design methods for multiband filters is to establish an analytical relation for the filter's response in terms of the filter topology. A major track in that manner is the use of frequency transformations.

The sequential application of the LPtoBP transformation generates even number of pass-bands after each sequential step. Nevertheless the band specifications of the multiband response cannot be directly controlled at each sequential step [16-18]. In [16] a direct LP to DB transformation is introduced in terms of the dual band specifications. By setting the corner frequencies of dual band characteristic, an LP to DB mapping function is generated in order to be applied on LP prototype to obtain the desired dual band form.

As illustrated in Fig. 1, a dual band gain characteristic is obtained [16] with the mapping function in (1). The associated frequency mapping diagram of the transformation

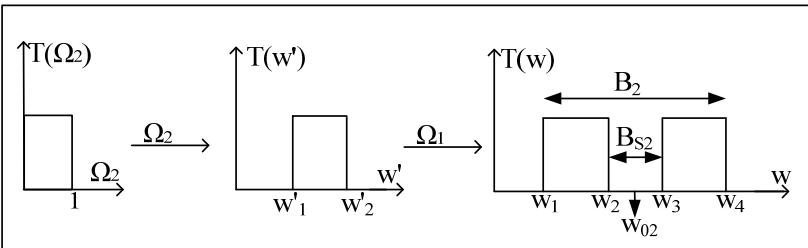

Fig. 1. Low-pass to dual band frequency mapping scheme.

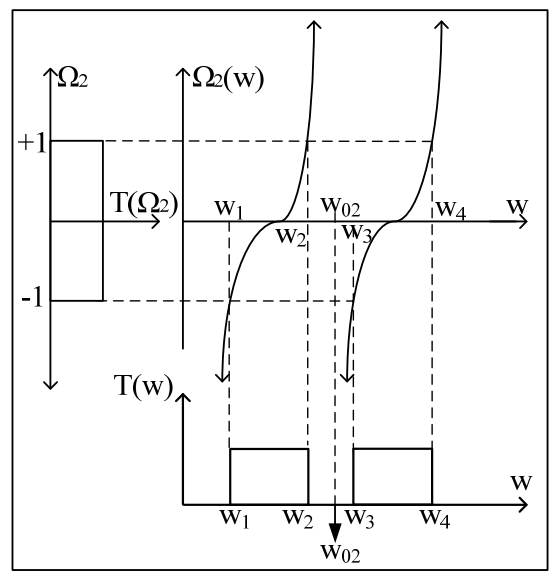

Fig. 2. LP to DB frequency mapping diagram.

is provided in Fig. 2.

$$
\begin{aligned}
& \Omega_{2}(w)=\frac{1}{B_{2}-B_{S 2}}\left(\frac{w^{2}-w_{02}^{2}}{w}\right)-\frac{B_{2} B_{S 2}}{B_{2}-B_{S 2}}\left(\frac{w}{w^{2}-w_{02}^{2}}\right), \\
& w_{02}=\sqrt{w_{1} w_{4}}=\sqrt{w_{2} w_{3}}, \\
& B_{2}=w_{4}-w_{1}, \quad B_{S 2}=w_{3}-w_{2} .
\end{aligned}
$$

Here, one can define an auxiliary variable such as;

$$
\Omega_{02}(w)=\left(\frac{w^{2}-w_{02}^{2}}{w}\right) .
$$

This variable is used for further simplification of the sequential LPtoBP transformation. It provides an overlook to see the general pattern of the sequential mapping functions.

Then the mapping function in (1) can be simplified by incorporating (2) as;

$$
\Omega_{2}(w)=\frac{1}{B_{2}-B_{S 2}} \Omega_{02}(w)-\frac{B_{2} B_{S 2}}{B_{2}-B_{S 2}} \frac{1}{\Omega_{02}(w)} .
$$

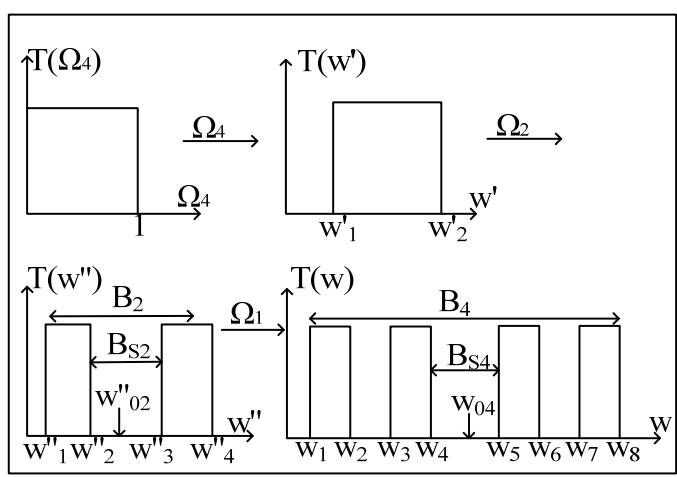

Fig. 3. Low-pass to quad-band transformation scheme. 


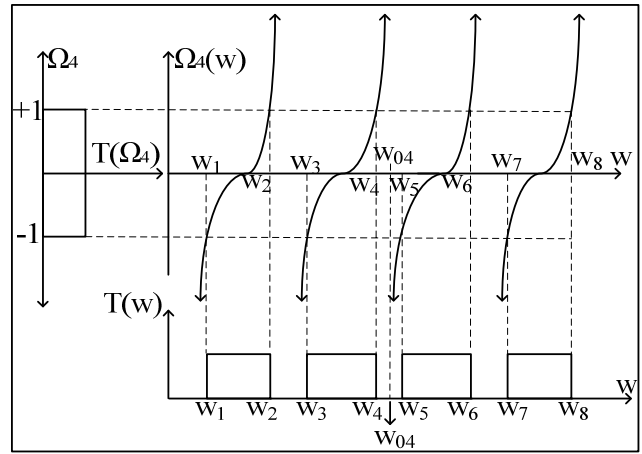

Fig. 4. Low-pass to quad-band frequency mapping diagram.

In [17] a quad band frequency transformation function is presented as another extension of the sequential LPtoBP mapping technique. As given in Fig. 3, a normalized LP prototype characteristic is mapped to QB gain characteristic in three sequential steps. The associated mapping diagram for the resultant mapping function in (4) is also presented in Fig. 4.

$$
\begin{aligned}
\Omega_{4}(w) & =\frac{1}{\left(B_{2}-B_{S 2}\right) B_{4}}\left(\frac{w^{2}-w_{04}^{2}}{w}\right)-\frac{1}{\left(B_{2}-B_{S 2}\right) B_{4}}\left(\frac{w}{w^{2}-w_{04}^{2}}\right) \\
& -\frac{B_{2} B_{S 2}}{B_{2}-B_{S 2}} \frac{1}{\frac{1}{B_{4}}\left(\frac{w^{2}-w_{04}^{2}}{w}\right)-B_{s 4}\left(\frac{w}{w^{2}-w_{04}^{2}}\right)} \\
w_{04} & =\sqrt{w_{1} w_{8}}=\sqrt{w_{2} w_{7}}=\sqrt{w_{3} w_{6}}=\sqrt{w_{4} w_{5}} .
\end{aligned}
$$

Here we use a similar auxiliary variable with different geometrical mean frequency as;

$$
\Omega_{04}(w)=\left(\frac{w^{2}-w_{04}^{2}}{w}\right) .
$$

This variable is incorporated in (4) then the mapping function in (4) is simplified as:

$$
\begin{aligned}
\Omega_{4}(w) & =\frac{1}{\left(B_{2}-B_{S 2}\right) B_{4}} \Omega_{04}(w)-\frac{1}{\left(B_{2}-B_{S 2}\right) B_{4}} \frac{1}{\Omega_{04}(w)} \\
& -\frac{B_{2} B_{S 2}}{B_{2}-B_{S 2}} \frac{1}{\frac{1}{B_{4}} \Omega_{04}(w)-B_{s 4} \frac{1}{\Omega_{04}(w)}} .
\end{aligned}
$$

As it can be interpreted from the mapping schemes in Fig. 1 and Fig. 3, each sequential step generates even ordered pass-bands. However a triple band transformation function can be derived by combining $\Omega_{4}$ and the $\Omega_{04}$ as:

$$
\begin{aligned}
\Omega_{3}(w)= & \Omega_{4}(w)+\frac{B_{S 4}}{\left(B_{2}-B_{S 2}\right)} \frac{1}{\Omega_{04}(w)}= \\
& \frac{1}{\left(B_{2}-B_{S 2}\right) B_{4}} \Omega_{04}(w)-\frac{B_{2} B_{S 2}}{B_{2}-B_{S 2}} \frac{1}{\frac{1}{B_{4}} \Omega_{04}(w)-B_{s 4} \frac{1}{\Omega_{04}(w)}} .
\end{aligned}
$$

Then the mapping function in (7) can be simplified as:

$$
\begin{aligned}
\Omega_{3}(w) & =\frac{1}{\left(B_{2}-B_{S 2}\right) B_{3}}\left(\frac{w^{2}-w_{03}^{2}}{w}\right)-\frac{1}{\left(B_{2}-B_{S 2}\right) B_{3}}\left(\frac{w}{w^{2}-w_{03}^{2}}\right) \\
& -\frac{B_{2} B_{S 2}}{B_{2}-B_{S 2}} \frac{1}{\frac{1}{B_{3}}\left(\frac{w^{2}-w_{03}^{2}}{w}\right)-w_{02}^{2} B_{3}\left(\frac{w}{w^{2}-w_{03}^{2}}\right)} .
\end{aligned}
$$

Here we use a similar auxiliary variable form as

$$
\Omega_{03}(w)=\left(\frac{w^{2}-w_{03}^{2}}{w}\right) .
$$

This variable is incorporated into (8) then the mapping function in (8) is simplified as:

$$
\begin{aligned}
\Omega_{3}(w)= & \frac{1}{\left(B_{2}-B_{S 2}\right) B_{3}} \Omega_{03}(w)-\frac{1}{\left(B_{2}-B_{S 2}\right) B_{3}} \frac{1}{\Omega_{03}(w)} \\
& -\frac{B_{2} B_{S 2}}{B_{2}-B_{S 2}} \frac{1}{\frac{1}{B_{3}} \Omega_{03}(w)-w_{02}^{2} B_{3} \frac{1}{\Omega_{03}(w)}} .
\end{aligned}
$$

The associated mapping diagram is given in Fig. 5 .

Even if the parametric form in (10) does not provide a direct determination of band characteristic of the triple band gain characteristic, it gives us an indication of the path to derive transformation function with odd ordered pass-bands by using weighted combinations of distinct steps of sequential LPtoBP transformations.

As it can be seen in the mapping diagrams in Fig. 2, Fig. 4 and Fig. 5, each asymptote maps the LP prototype response to its associated pass-band at the overall multiband characteristic. These asymptotes directly control the edge frequencies of each pass-band. This result and the forms of the transformation equations in (3), (6) and (10) lead us to propose a multiband mapping function as a generalized extension of the sequential LPtoBP transformations. To obtain multiband gain characteristic with assigned number of pass-bands, there must be a suitable multiband mapping function whose mapping diagram must have assigned number of asymptotes which correspond to the multiple of pass-bands.

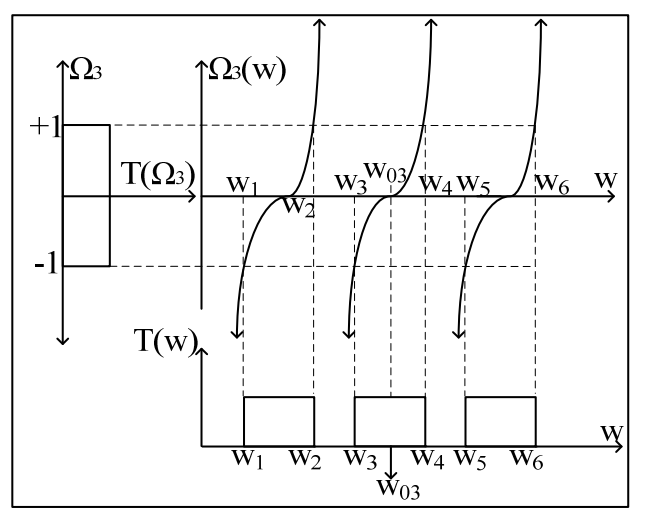

Fig. 5. Low-pass to triple band frequency mapping diagram. 


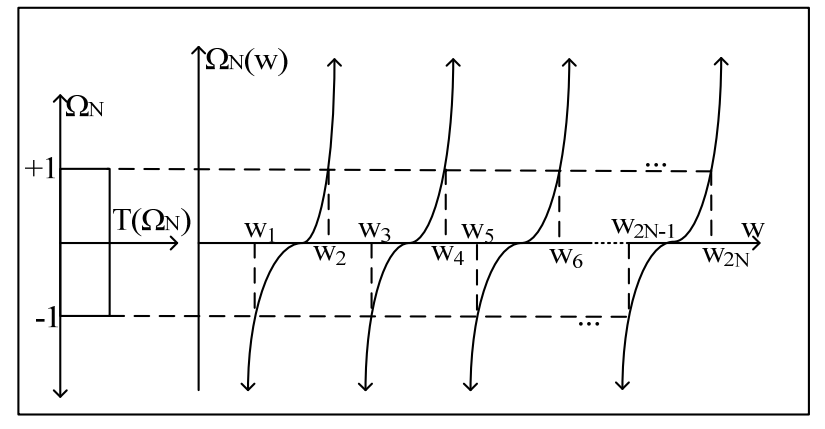

Fig. 6. Low pass to multiband frequency mapping diagram.

Considering the mapping diagram in Fig. 6, if the asymptotes are placed on the mapping diagram at assigned places with assigned slopes, then the number of pass-bands and their characteristics can be controlled.

The normalized LP characteristic in $\Omega_{N}$ domain is mapped to multiband gain characteristic in $w$ domain. Here we propose the following generalized mapping function in (11) to generate such a mapping diagram.

$$
\begin{aligned}
& \Omega_{0 N}(w)=\left(\frac{w^{2}-w_{0 N}^{2}}{w}\right), \\
& \Omega_{N}(w)=a_{1} \Omega_{0 N}(w)-\frac{1}{a_{2} \Omega_{0 N}(w)-\frac{1}{a_{3} \Omega_{0 N}(w)-\cdot-\frac{1}{a_{N} \Omega_{0 N}(w)}}} .
\end{aligned}
$$

Here $N$ represents the number of pass-bands, and the passband edge frequencies are distributed around the geometrical center frequency of $w_{0 N}$. The ripple characteristic of the LP prototype response is preserved through the transformation process unless bandwidths are scaled in accordance with the associated asymptote for each pass-band. Thus if $n$ degree LP prototype is chosen then the resultant $N$ band filter will be degree of $N(2 n)$. Since this mapping function is based on sequential LPtoBP mapping, the relations between the band edge frequencies is inherently preserved as in [16] and [17]. The following parametric relations should be satisfied as:

$$
\begin{aligned}
& w_{0}=\sqrt{w_{1} w_{2 N}}=\sqrt{w_{2} w_{2 N-1}} \ldots, \\
& w_{1}<w_{2}<\ldots<w_{0}<\ldots<w_{2 N-1}<w_{2 N} .
\end{aligned}
$$

In the proposed LP to multiband transformation, the number of $N+1$ edge frequencies of the multiband response can be assigned independently, the remaining corner frequencies are determined under the geometric symmetry constraint of the mapping in (12). In this case, by selecting any of the $\mathrm{N}+1$ band edge frequencies, the mapping function can be derived in terms of $a_{N}$ coefficients. These coefficients determine the slope characteristics of the asymptotes at the mapping diagram. Moreover the mapping diagram in Fig. 6 displays that at corner frequencies $\left|\Omega_{N}(w)\right|=1$. Setting the corner frequencies of multiband response will provide us number of $2 N$ equations with $N$ unknown $a_{N}$ parameters. By selecting any set of $N$ equations from those $2 N$ equations and using a numerical solver, these $a_{N}$ coefficients can be calculated.

For example, if the normalized cut-off frequencies are defined as $w_{1}=1, w_{2}=1.5, w_{3}=2$, and then $w_{4}=3$ as indicated in (12). The associated $a_{N}$ parameters are calculated by solving the following equations as below:

$$
\begin{aligned}
& \Omega_{02}(w)=\left(\frac{w^{2}-w_{02}^{2}}{w}\right), \quad w_{02}=\sqrt{w_{1} w_{4}}=1.732, \\
& \Omega_{2}(w)|=| a_{1} \Omega_{02}(w)-\left.\frac{1}{a_{2} \Omega_{02}(w)}\right|_{w=\left\{w_{1}, w_{2}, w_{3}, w_{4}\right\}}=1 .
\end{aligned}
$$

By choosing any two sets of those four equations these parameters can be calculated such as:

$$
\mid \begin{aligned}
& \left|a_{1}\left(\frac{w_{1}^{2}-3}{w_{1}}\right)-\frac{1}{a_{2}\left(\frac{w_{1}^{2}-3}{w_{1}}\right)}\right|=1, \\
& \left|a_{1}\left(\frac{w_{3}^{2}-3}{w_{3}}\right)-\frac{1}{a_{2}\left(\frac{w_{3}^{2}-3}{w_{3}}\right)}\right|=1 .
\end{aligned}
$$

These parameters are calculated as $a_{1}=0.667$, $a_{2}=1.5$. The mapping function is derived as;

$$
\Omega_{2}(w)=0.667 \Omega_{02}(w)-\frac{1}{1.5 \Omega_{02}(w)} .
$$

The generated mapping function is applied on the $11^{\text {th }}$ degree equal ripple Chebyshev LP prototype [1] and the resulting dual band gain and return loss characteristic are as shown in Fig. 7. This filter is $44^{\text {th }}$ degree (i.e. $\left.2(2 \times 11)\right)$ as a result of application of mapping function on filter transfer function.

Considering the following triple band mapping function example, the normalized cut-off frequencies are assigned as $w_{1}=0.85, w_{2}=1.15, w_{6}=3, w_{3}=1.5$, and the

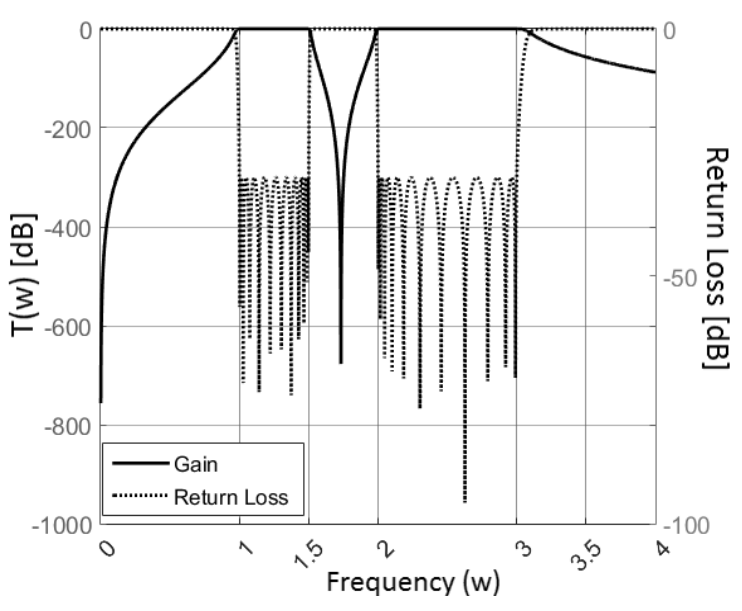

Fig. 7. Gain and return loss response of dual band characteristic produced with LP to multiband. 
remaining corner frequencies are calculated as $w_{1}=1.7$, $w_{5}=2.2174$ by using the relation in (12). The associated $a_{N}$ parameters are calculated by solving the following equation as:

$$
\begin{aligned}
& \Omega_{03}(w)=\left(\frac{w^{2}-w_{03}^{2}}{w}\right), \quad w_{03}=\sqrt{w_{1} w_{6}}=1.5969, \\
& \left|\Omega_{3}(w)\right|=\mid \begin{array}{l}
a_{1} \Omega_{03}(w)-\left.\frac{1}{a_{2} \Omega_{03}(w)-\frac{1}{a_{3} \Omega_{03}(w)}}\right|_{w=\left\{w_{1}, w_{2}, w_{3}, w_{4}, w_{5}, w_{6}\right\}}=1
\end{array}
\end{aligned}
$$

By choosing any three sets of those six equations these parameters can be calculated as;

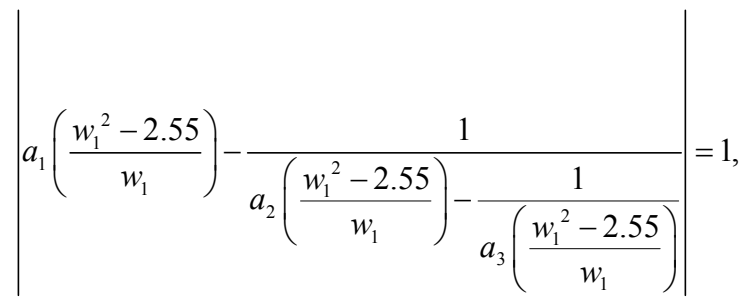

$$
\begin{aligned}
& \left|a_{1}\left(\frac{w_{3}{ }^{2}-2.55}{w_{3}}\right)-\frac{1}{a_{2}\left(\frac{w_{3}{ }^{2}-2.55}{w_{3}}\right)-\frac{1}{a_{3}\left(\frac{w_{3}{ }^{2}-2.55}{w_{3}}\right)}}\right|=1 \text {, } \\
& \left|a_{1}\left(\frac{w_{5}{ }^{2}-2.55}{w_{5}}\right)-\frac{1}{a_{2}\left(\frac{w_{5}{ }^{2}-2.55}{w_{5}}\right)-\frac{1}{a_{3}\left(\frac{w_{5}{ }^{2}-2.55}{w_{5}}\right)}}\right|=1
\end{aligned}
$$

These parameters are found as $a_{1}=0.7797, a_{2}=$ $0.7455, a_{3}=3.7486$. The mapping function is derived as

$$
\Omega_{3}(w)=0.7797 \Omega_{03}(w)-\frac{1}{0.7455 \Omega_{03}(w)-\frac{1}{3.7486 \Omega_{03}(w)}} .
$$

This mapping function is applied on the $9^{\text {th }}$ degree equal ripple Chebyshev LP prototype [1] and the resulting triple band gain and return loss characteristic are shown in Fig. 8. This filter turns out to be the $54^{\text {th }}$ degree (i.e. $3(2 \times 9))$ as a result of application of the mapping function on the prototype filter transfer function.

In this method, the selectivity of the multiband filter inherently depends on complexity of the chosen LP prototype network and also on assigned cut-off frequencies of the multiband response. Higher degree LP prototypes will provide more selective multiband filters with same cut-off frequencies. The cut-off frequencies of the multiband filter can be chosen arbitrarily under the constraint in (12). If a lower degree LP prototype is chosen, and the pass-bands

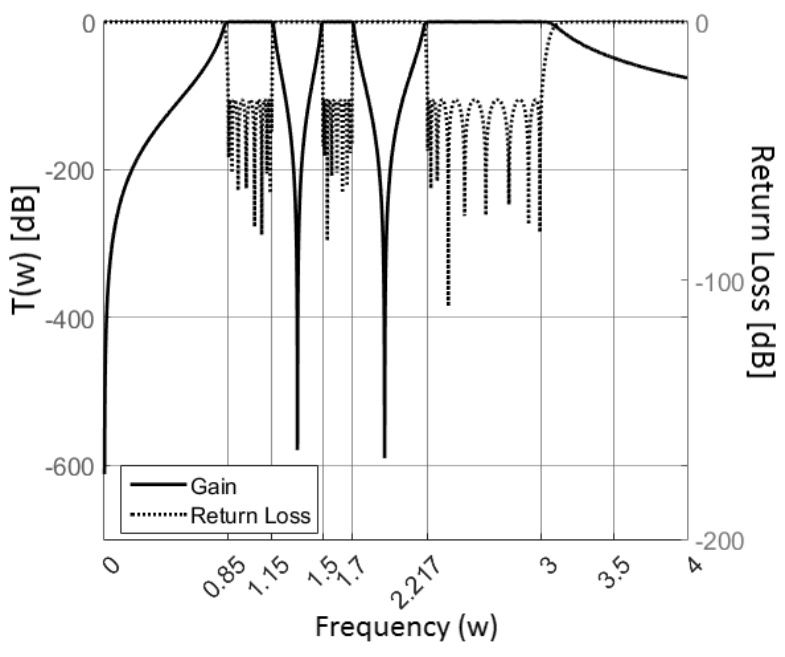

Fig. 8. Gain and return loss response of triple band characteristic produced with LP to multiband.

are placed closely with each other, this may cause signal crosstalk due to limited attenuation between pass-bands [15].

\section{Lumped Synthesis of Transformed Reactance}

The synthesis of multiband network is obtained by the successive application of the determined multiband mapping function on the chosen LP prototype network. Each reactive element of the LP prototype network is mapped to multiband correspondences by using the multiband mapping function as a new frequency variable. Thus the one port synthesis of each transformed element is achieved.

Here we assume the prototype LP network is only composed of lossless reactive elements. Given a capacitor or an inductor, the multiband transformed impedance or admittance can be derived as:

$Z_{\mathrm{L}}\left(\Omega_{N}(w)\right)=\mathrm{j} \Omega_{N}(w) L$

$=\mathrm{j}\left(a_{1}\left(\frac{w^{2}-w_{0 N}^{2}}{w}\right)-\frac{1}{a_{2}\left(\frac{w^{2}-w_{0 N}^{2}}{w}\right)-\ddots \frac{1}{a_{N}\left(\frac{w^{2}-w_{0 N}^{2}}{w}\right)}}\right) L$,

$Y_{\mathrm{C}}\left(\Omega_{N}(w)\right)=\mathrm{j} \Omega_{N}(w) C$

$=\mathrm{j}\left(a_{1}\left(\frac{w^{2}-w_{0 N}^{2}}{w}\right)-\frac{1}{a_{2}\left(\frac{w^{2}-w_{0 N}^{2}}{w}\right)-\ddots \frac{1}{a_{N}\left(\frac{w^{2}-w_{0 N}^{2}}{w}\right)}}\right) C$.

One port synthesis of the admittance or impedance above is synthesized as cascaded series and/or shunt reso- 
nators [10]. The element values of the each resonator are deduced directly from the mapped reactance function in (19) and (20).

Considering the dual-band mapping function as:

$$
\Omega_{2}(w)=a_{1}\left(\frac{w^{2}-w_{02}^{2}}{w}\right)-\frac{1}{a_{2}\left(\frac{w^{2}-w_{02}^{2}}{w}\right)} .
$$

The dual band transformed impedance of inductor is derived as:

$$
\begin{aligned}
& Z_{\mathrm{L}}\left(\Omega_{2}(w)\right)=\mathrm{j} \Omega_{2}(w) L \\
& =\mathrm{j} a_{1} L w+\frac{1}{\mathrm{j} w \frac{1}{a_{1} L w_{02}^{2}}}+\frac{1}{\mathrm{j} w \frac{a_{2}}{L}+\frac{1}{\mathrm{j} w \frac{L}{a_{2} w_{02}^{2}}}} \\
& =\mathrm{j} w L_{1}+\frac{1}{\mathrm{j} w C_{1}}+\frac{1}{\mathrm{j} w C_{2}+\frac{1}{\mathrm{j} w L_{2}}}, \\
& L_{1}=a_{1} L, \quad C_{1}=\frac{1}{a_{1} L w_{02}^{2}}, \quad C_{2}=\frac{a_{2}}{L}, \quad L_{2}=\frac{L}{a_{2} w_{02}^{2}} .
\end{aligned}
$$

The dual band transformed admittance of capacitor is derived as:

$$
\begin{aligned}
& Y_{\mathrm{C}}\left(\Omega_{2}(w)\right)=\mathrm{j} \Omega_{2}(w) C \\
& =\mathrm{j} a_{1} C w+\frac{1}{\mathrm{j} w \frac{1}{a_{1} C w_{02}^{2}}}+\frac{1}{\mathrm{j} w \frac{a_{2}}{C}+\frac{1}{\mathrm{j} w \frac{C}{a_{2} w_{02}^{2}}}} \\
& =\mathrm{j} w C_{1}+\frac{1}{\mathrm{j} w L_{1}}+\frac{1}{\mathrm{j} w L_{2}+\frac{1}{\mathrm{j} w C_{2}}}, \\
& C_{1}=a_{1} C, \quad L_{1}=\frac{1}{a_{1} C w_{02}^{2}}, \quad L_{2}=\frac{a_{2}}{C}, \quad C_{2}=\frac{C}{a_{2} w_{02}^{2}} .
\end{aligned}
$$

The lumped element synthesis of the dual band transformed impedance and admittance for an inductor and a capacitor are illustrated in Fig. 9a and Fig. 9b, respectively.

Considering the triple-band mapping function as:

$$
\Omega_{3}(w)=a_{1}\left(\frac{w^{2}-w_{03}^{2}}{w}\right)-\frac{1}{a_{2}\left(\frac{w^{2}-w_{03}^{2}}{w}\right)-\frac{1}{a_{3}\left(\frac{w^{2}-w_{03}^{2}}{w}\right)} .}
$$

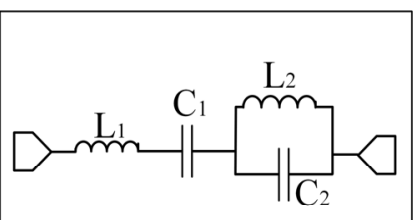

(a)

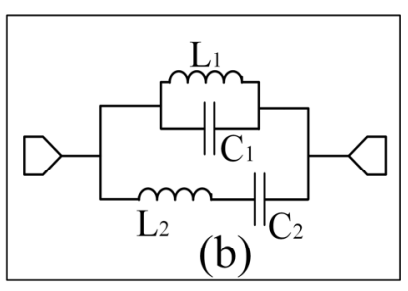

(b)
Fig. 9. a) Transformed inductor for dual band mapping. b) Transformed capacitor for dual band mapping.

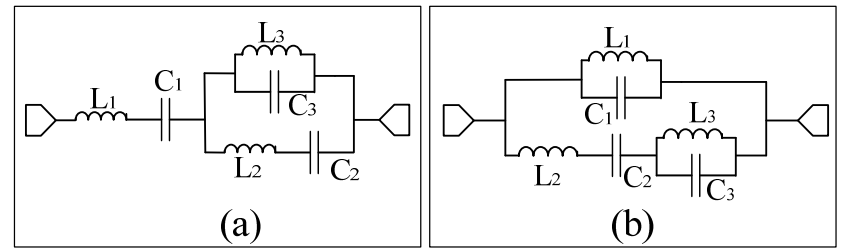

Fig. 10. a) Transformed inductor for triple band mapping. b) Transformed capacitor for triple band mapping.

The triple band transformed impedance of inductor is derived as:

$$
\begin{aligned}
& Z_{\mathrm{L}}\left(\Omega_{3}(w)\right)=\mathrm{j} \Omega_{3}(w) L \\
& =\mathrm{j} w a_{1} L+\frac{1}{\mathrm{j} w \frac{1}{a_{1} L w_{03}^{2}}}+\frac{1}{\mathrm{j} w \frac{a_{2}}{L}+\frac{1}{\mathrm{j} w \frac{L}{a_{2} w_{03}^{2}}}+\frac{1}{\mathrm{j} w a_{3} L+\frac{1}{\mathrm{j} w \frac{1}{a_{3} L w_{03}^{2}}}}} \\
& =\mathrm{j} w L_{1}+\frac{1}{\mathrm{j} w C_{1}}+\frac{1}{\mathrm{j} w C_{2}+\frac{1}{\mathrm{j} w L_{2}}+\frac{1}{\mathrm{j} w L_{3}+\frac{1}{\mathrm{j} w C_{3}}}}, \\
& L_{1}=a_{1} L, \quad C_{1}=\frac{1}{a_{1} L w_{03}^{2}}, \quad L_{2}=\frac{L}{a_{2} w_{03}^{2}}, \quad C_{2}=\frac{a_{2}}{L}, \\
& L_{3}=a_{3} L, \quad C_{3}=\frac{1}{a_{3} L w_{03}^{2}} .
\end{aligned}
$$

The triple band transformed admittance of capacitor is derived as:

$$
\begin{aligned}
& Y_{\mathrm{C}}\left(\Omega_{3}(w)\right)=\mathrm{j} \Omega_{3}(w) C \\
& =\mathrm{j} w a_{1} C+\frac{1}{\mathrm{j} w \frac{1}{a_{1} C w_{03}^{2}}}+\frac{1}{\mathrm{j} w \frac{a_{2}}{C}+\frac{1}{\mathrm{j} w \frac{C}{a_{2} w_{03}^{2}}}+\frac{1}{\mathrm{j} w a_{3} C+\frac{1}{\mathrm{j} w \frac{1}{a_{3} C w_{03}^{2}}}}} \\
& =\mathrm{j} w C_{1}+\frac{1}{\mathrm{j} w L_{1}}+\frac{1}{\mathrm{j} w L_{2}+\frac{1}{\mathrm{j} w C_{2}}+\frac{1}{\mathrm{j} w C_{3}+\frac{1}{\mathrm{j} w L_{3}}}}, \\
& C_{1}=a_{1} C, \quad L_{1}=\frac{1}{a_{1} C w_{03}^{2}}, \quad C_{2}=\frac{C}{a_{2} w_{03}^{2}}, \quad L_{2}=\frac{a_{2}}{C}, \\
& C_{3}=a_{3} C, \quad L_{3}=\frac{1}{a_{3} C w_{03}^{2}} \text {. }
\end{aligned}
$$

The lumped element synthesis of the triple band transformed impedance and admittance for an inductor and a capacitor are illustrated in Fig. 10a and Fig. 10b, respectively.

As a result, the mapping function directly determines the topology of the multiband network and their element values.

\section{Dual Band Filter Design for GSM900 and GSM1800/UMTS}

In this section, the dual band filter design in [18] is re-considered by employing the new method. Here, the aim 
of this process is the validation of the relation between the proposed mapping function and the sequential LP to DB mapping function.

The band specifications in [18] are preserved therefore the normalized parameters of the mapping function are $w_{1}=0.85, w_{2}=1.15, w_{4}=2.3$ and the last edge frequency is determined using (12) as $w_{3}=1.7$. The geometric center frequency is calculated as $w_{0}=1.398$ by using (12).

The mapping function in [18] is derived using (1) as:

$$
\begin{aligned}
& \Omega_{2}(w)=\frac{1}{B_{2}-B_{S}}\left(\frac{w^{2}-w_{02}^{2}}{w}\right)-\frac{B_{2} B_{S}}{B_{2}-B_{S}}\left(\frac{w}{w^{2}-w_{02}^{2}}\right), \\
& B_{2}=w_{4}-w_{1}=1.45, \quad B_{S}=w_{3}-w_{2}=0.55, \\
& \Omega_{2}(w)=1.111\left(\frac{w^{2}-1.955}{w}\right)-0.8861\left(\frac{w}{w^{2}-1.955}\right), \\
& \Omega_{2}(w)=1.111\left(\frac{w^{2}-1.955}{w}\right)-\frac{1}{1.1285\left(\frac{w^{2}-1.955}{w}\right)} .
\end{aligned}
$$

If we apply the new multiband mapping function in (11) for the assigned band characteristic then the mapping function is derived as:

$$
\begin{aligned}
& \Omega_{02}(w)=\left(\frac{w^{2}-w_{02}^{2}}{w}\right), \quad w_{02}=\sqrt{w_{1} w_{4}}=1.398, \\
& \left|\Omega_{2}(w)\right|=\left|a_{1} \Omega_{02}(w)-\frac{1}{a_{2} \Omega_{02}(w)}\right|_{w=\left\{w_{1}, w_{2}, w_{3}, w_{4}\right\}}=1 .
\end{aligned}
$$

The two sets of equations are solved and the unknown parameters are calculated as $a_{1}=1.111, a_{2}=1.285$.

Eventually the mapping function is derived as:

$$
\Omega_{2}(w)=1.111 \Omega_{02}(w)-\frac{1}{1.285 \Omega_{02}(w)} .
$$

As seen in (27) and in (29), the mapping functions are identical. This emphasizes that the proposed technique is a generalized form of the classical sequential LPtoBP mapping approach.

\section{Triple Band Filter Design Example}

In this section, a triple band filter design is considered. The normalized cut-off frequencies are chosen as $w_{1}=0.45, w_{2}=0.75, w_{3}=0.99, w_{6}=2.7$, then the remaining corner frequencies are calculated as $w_{4}=1.2273, w_{5}=1.62$ by using (12). The associated $a_{N}$ parameters are calculated by solving the 3 equations from those six equations below:

$$
\begin{aligned}
& \Omega_{03}(w)=\left(\frac{w^{2}-w_{03}^{2}}{w}\right), \quad w_{03}=\sqrt{w_{1} w_{6}}=1.1023, \\
& \left|\Omega_{3}(w)\right|=\left|a_{1} \Omega_{03}(w)-\frac{1}{a_{2} \Omega_{03}(w)-\frac{1}{a_{3} \Omega_{03}(w)}}\right|_{w=\left\{w_{1}, w_{2}, w_{3}, w_{4}, w_{5}, w_{6}\right\}}=1
\end{aligned}
$$

These parameters are found as $a_{1}=0.6183, a_{2}=$ 1.2043, $a_{3}=2.8913$. The mapping function is derived as:

$$
\Omega_{3}(w)=0.6183 \Omega_{03}(w)-\frac{1}{1.2043 \Omega_{03}(w)-\frac{1}{2.8913 \Omega_{03}(w)}} .
$$

This mapping function is applied on the $3^{\text {rd }}$ degree equal ripple Chebyshev LP prototype as given in Fig. 11.

The triple band filter is achieved by applying the mapping function in (31) to each reactive component of the LP prototype network.

Since the explicit network topologies of the triple band transformed inductor and capacitor are given in Fig. 10, the transformed triple band filter network is synthesized as in Fig. 12. The gain and return loss characteristic of the triple band filter are given in Fig. 13.

Using the realistic element models and performing the EM simulation in ADS momentum environment, the realizable version of the filter network is generated. To do this, first the paths and soldering pads are designed to connect lumped elements to each other. But these structures inevitably affect the performance by acting as distributed elements and generate extra inductances and capacitances. Therefore to approach to the similar results with ideal lumped design, all lumped components are tuned and changed with their realistic counterparts (for capacitances GJM15 series from Murata and for inductances 06CS series from Coilcraft are used), which include the parasitics and loss of realistic models. Only C5 and C7 in Fig. 12 are realized as open radial stubs for convenience in production

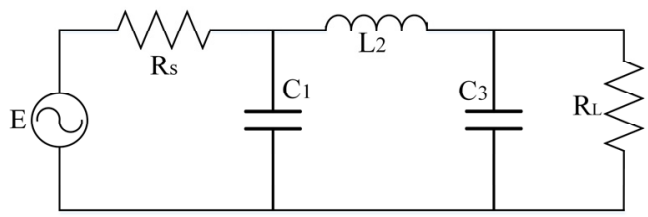

Fig. 11. The $3^{\text {rd }}$ degree equal ripple Chebyshev LP prototype network $(\mathrm{Rs}=50 \Omega, \mathrm{C} 1=15.98 \mathrm{pF}, \mathrm{L} 2=29.58 \mathrm{nH}$, $\mathrm{C} 3=15.98 \mathrm{pF}, \mathrm{RL}=50 \Omega$ )

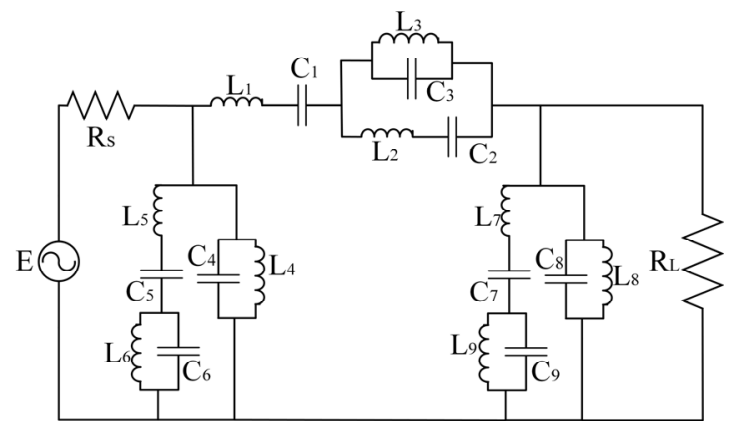

Fig. 12. Transformed triple band filter network ( $\mathrm{L} 1=5.5 \mathrm{nH}$ $\mathrm{L} 2=25.674 \mathrm{nH}, \mathrm{L3}=6.07 \mathrm{nH}, \mathrm{L} 4=7.024 \mathrm{nH}, \mathrm{L} 5=$ $6.36 \mathrm{nH}, \mathrm{L} 6=1.5 \mathrm{nH}, \mathrm{L} 7=6.36 \mathrm{nH}, \mathrm{L} 8=7.024 \mathrm{nH}$, $\mathrm{L} 9=1.5 \mathrm{nH}, \quad \mathrm{C} 1=3.8 \mathrm{pF}, \quad \mathrm{C} 2=0.812 \mathrm{pF}, \quad \mathrm{C} 3=$ $3.44 \mathrm{pF}, \mathrm{C} 4=2.97 \mathrm{pF}, \mathrm{C} 5=3.28 \mathrm{pF}, \mathrm{C} 6=13.96 \mathrm{pF}$, $\mathrm{C} 7=3.28 \mathrm{pF}, \quad \mathrm{C} 8=2.97 \mathrm{pF}, \quad \mathrm{C} 9=13.96 \mathrm{pF}, \quad \mathrm{RL}=$ $\mathrm{Rs}=50 \Omega$ ). 


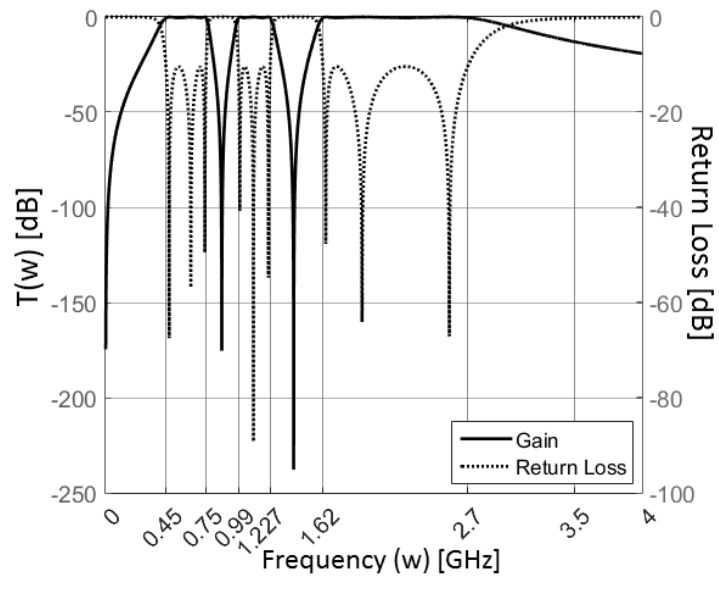

Fig. 13. Gain and return loss response of the triple band filter.

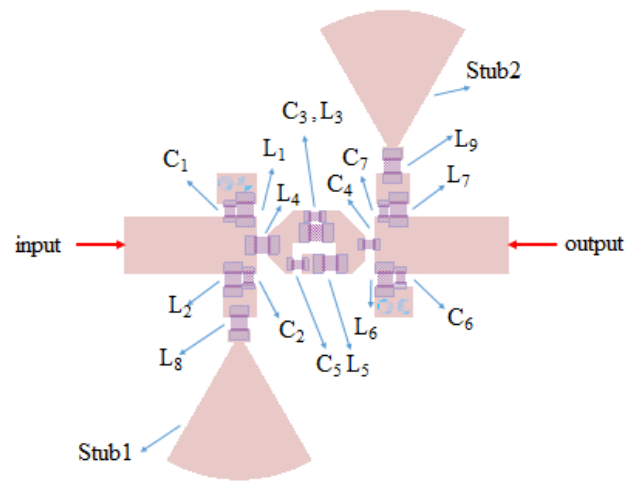

Fig. 14. Layout of the implemented triple band filter.

\begin{tabular}{|c|c|}
\hline Element & Value \\
\hline $\mathrm{C} 1, \mathrm{C} 6$ & $0.6 \mathrm{pF}$ \\
\hline $\mathrm{L} 1, \mathrm{~L} 6, \mathrm{~L} 8, \mathrm{~L} 9$ & $10 \mathrm{nH}$ \\
\hline $\mathrm{C} 2, \mathrm{C} 7$ & $5.6 \mathrm{pF}$ \\
\hline $\mathrm{L} 2, \mathrm{~L} 7$ & $3.9 \mathrm{nH}$ \\
\hline Stub1, Stub2 & $\mathrm{L}=7 \mathrm{~mm}, \mathrm{Ang}=60 \mathrm{Deg}, \mathrm{Wi}=0.5 \mathrm{~mm}$ \\
\hline $\mathrm{C} 3$ & $3.0 \mathrm{pF}$ \\
\hline $\mathrm{L} 3$ & $5.1 \mathrm{nH}$ \\
\hline $\mathrm{C} 4$ & $3.6 \mathrm{pF}$ \\
\hline $\mathrm{L} 4$ & $3.3 \mathrm{nH}$ \\
\hline $\mathrm{C} 5$ & $0.8 \mathrm{pF}$ \\
\hline $\mathrm{L} 5$ & $23 \mathrm{nH}$ \\
\hline PCB & FR4, $1.6 \mathrm{~mm}, \varepsilon_{\mathrm{r}}=4.8$ \\
\hline
\end{tabular}

Tab. 1. Component values of the prototyped filter. (a)

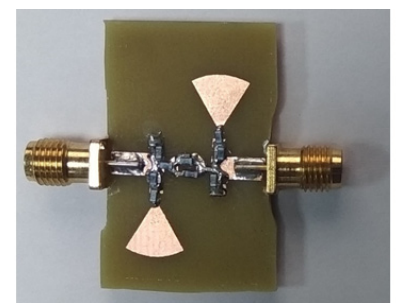

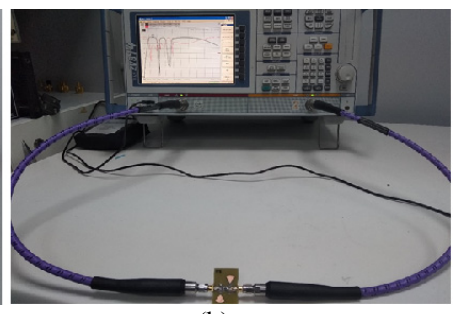

(b)
Fig. 15. (a) Implemented filter, (b) measurement set-up.

[19]. After this process, overall performance of the filter network is optimized and the obtained best results are used for the production phase. The layout of the prototyped filter is given in Fig. 14. The element values are given in Tab. 1 .

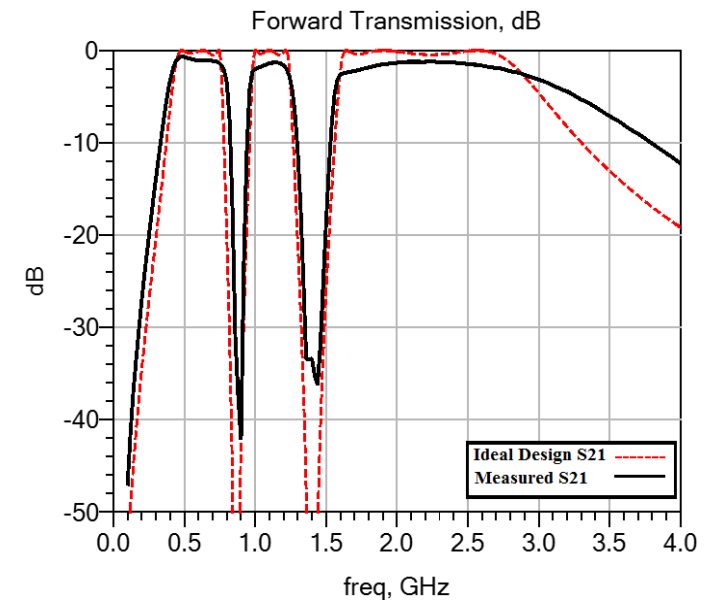

Fig. 16. Gain response of the triple band filter.

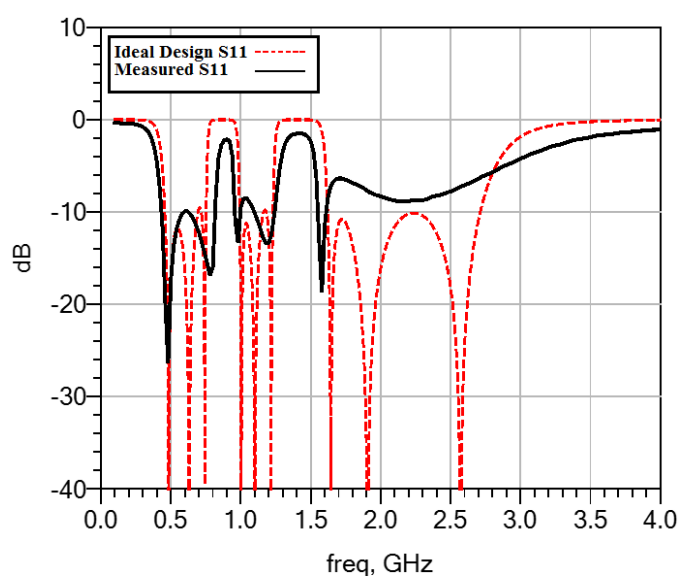

Fig. 17. Return loss response of the triple band filter.

Manufactured mixed element prototype of the triple band filter and the measurement set-up are given in Fig. 15a and Fig. 15b, respectively.

The implemented filter is measured by using Rohde\&Schwarz ZVB14 network analyzer. Comparison of S21 and S11 responses of the ideal lumped model and the implemented filter are given in Fig. 16 and Fig. 17, respectively.

The performances of the designed ideal lumped triple band filter and the implemented mixed triple band filter are consistent with each other. The observed difference between the ideal and the measurement performances are due to element approximations in implemented filter, EM models inconsistencies, component tolerances, dielectric losses, and also measurement inaccuracies.

\section{Conclusion}

In this paper, a new generalized multiband frequency mapping function and its usage in multiband filter design is introduced. The constraints of the mapping function are elaborated. The proposed mapping function is a generalized form of the well-known sequential LPtoBP transformation. 
A direct relation between LP and multiband responses is provided using the proposed multiband mapping function. The applications of the multiband mapping function for different number of pass-bands are presented. The synthesis of multiband filter is obtained by using multiband element mapping of a chosen prototype network. The explicit transformed network topologies are presented for dual band and triple band mapping functions. A triple band filter design and its implementation is presented. The measurement results showed that the performance of the implemented filter is consistent with the aimed triple band design specifications.

\section{References}

[1] CRNOJEVIC-BENGIN, V. (Ed.) Advances in Multi-Band Microstrip Filters. Cambridge (UK): Cambridge University Press, 2015. ISBN: 9781139976763

[2] ABDELNOUR, A., LAZARO, A., VILLARINO, R., et al. Passive harmonic RFID system for buried assets localization. Sensors, 2018, vol. 18, no. 11, p. 1-21. DOI: $10.3390 /$ s18113635

[3] KUMAR, G. J. R., SHAJI, K. S. Design and analysis of multiband OFDM system over ultra wide band channels. Indian Journal of Computer Science and Engineering, 2013, vol. 4, no. 1, p. 69-73. ISSN : 0976-5166

[4] KOIKE, Y. Difference between Wide-band and Narrow-band Radio Module. Technical information. Circuit Design, Inc., 15 Dec. 2015, p. 1-4.

[5] YILDIZ, S., AKSEN, A., YARMAN, B. S. Multiband and concurrent matching network design via Brune sections. In The 24th IEEE International Conference on Electronics, Circuits and Systems (ICECS). Batumi (Georgia), 2017, p. 90-93. DOI: 10.1109/ICECS.2017.8292058

[6] YILDIZ, S., AKSEN, A., YARMAN, B. S. A numerical approach for the design of matching networks consisting of Brune sections based on Fujisawa constraints. In The 18th Mediterranean Microwave Symposium (MMS2018). Istanbul (Turkey), 2018, p. 171-174. DOI: 10.1109/MMS.2018.8611905

[7] YILDIZ, S., AKSEN, A., YARMAN, B. S. Real frequency design of multiband matching networks with mixed lumped-distributed elements and Foster resonance sections. In The 18th Mediterranean Microwave Symposium (MMS2018). Istanbul (Turkey), 2018, p. 187-190. DOI: 10.1109/MMS.2018.8611994

[8] FUJiMOTO, H., MURAKAMI, K., KITAZAWA, S. Equivalent circuits and transmission zeros of the coupled square-loop resonator. IEICE Electronics Express, 2007, vol. 4, no. 18, p. 575-581. DOI: $10.1587 /$ elex.4.575

[9] ATILlA, D. C., YARMAN, B. S., AKSEN, A., et al. Computer aided Darlington synthesis of an all-purpose immittance function. Istanbul University - Journal of Electrical and Electronics Engineering, 2016, vol. 16, no. 1, p. 2027-2037.

[10] YOULA, D. C. Theory and Synthesis of Linear Passive TimeInvariant Networks. 1st ed. Cambridge University Press; 2015. (p. 271-350). ISBN: 9781316403105

[11] MACCHIAREllA, G., TAMIAZZO, S. Design techniques for dual-passband filters. IEEE Transactions on Microwave Theory and Techniques, 2005, vol. 53, no. 11, p. 3265-327. DOI: 10.1109/TMTT.2005.855749

[12] DUTTA ROY, S. C. Network design for multiple frequency impedance matching by the frequency transformation technique.
IETE Journal of Research, 2014, vol. 59, no. 6, p. 698-703. DOI: 10.4103/0377-2063.126966

[13] BRAND, T. G., MEYER, P., GESCHKE, R. H. Designing multiband coupled-resonator filters using reactance transformations, International Journal of $R F$ and Microwave Computer-Aided Engineering, 2015, vol. 25, no. 1, p. 81-92. DOI: 10.1002/mmce. 20826

[14] GARCÍA-LAMPÉREZ, A., SALAZAR-PALMA, M. Single-band to multiband frequency transformation for multiband filters. IEEE Transactions on Microwave Theory and Techniques, 2011, vol. 59, no. 12, p. 3048-3058. DOI: 10.1109/TMTT.2011.2170579

[15] YILDIZ, S., AKSEN, A., YARMAN, B. S. Dual band filter design using real frequency technique and frequency transformation. Istanbul University - Journal of Electrical and Electronics Engineering, 2017, vol. 17, no. 2, p. 3343-3350.

[16] AKSEN, A., YILDIZ, S., YARMAN, S. B. A frequency transformation based real frequency design approach for dual-band matching. In 2017 International Symposium on Signals, Circuits and Systems (ISSCS). Iasi (Romania), 2017, p. 1-4. DOI: 10.1109/ISSCS.2017.8034909

[17] YILDIZ, S., AKSEN, A., YARMAN, B. S. Quad-band matching network design with real frequency technique employing frequency transformation. In The IEEE First Ukraine Conference on Electrical and Computer Engineering (UKRCON-2017). Kiev (Ukraine), 2017, p. 143-147. DOI: 10.1109/UKRCON.2017.8100462

[18] YILDIZ, S., AKSEN, A., KILINÇ, S., YARMAN, B. S. Dual band filter design for GSM bands using frequency mapping and real frequency technique. In The 18th Mediterranean Microwave Symposium (MMS2018). Istanbul (Turkey), 2018, p. 164-167. DOI: $10.1109 /$ MMS.2018.8612080

[19] RHEA, R. W. HF Filter Design and Computer Simulation. Scitech Publishing, 1994. ISBN: 1884932258

\section{About the Authors ...}

Serkan YILDIZ (corresponding author) received his B.Sc. degree in Electrical and Electronics Engineering from Istanbul University, Turkey, in 2009 and M.Sc. degree from Istanbul Technical University, Turkey, in 2011, and he is currently working towards the Ph.D. degree at Electronics Engineering of Isik University. He was with the Electronics and Communication Engineering Department of Istanbul Technical University between 2013-2017. He is with The Scientific and Technological Research Council of Turkey since 2017. His research interests include RF and microwave filter design, matching network design and network synthesis.

Ahmet AKSEN received B.Sc. and M.Sc. degrees in Electrical and Electronics Engineering from the Middle East Technical University, Ankara, Turkey in 1981 and 1985, respectively and the Ph.D. degree from the Ruhr University, Bochum, Germany in 1994. Currently, he is the chairman of the Department of Electrical and Electronics Engineering of Işık University. His research interests are in the area of multivariable network theory and in the design of microwave filters, broadband matching networks and amplifiers. 
Sedat KILINC received his B.Sc. and M.Sc. degrees in Electronics and Communications Engineering from Yildiz Technical University and Technical University of Istanbul in 2012 and 2015. He is currently studying towards his Ph.D. degree in Technical University of Istanbul. He has also been studying as a research assistant in Istanbul University since 2012 . His research interests are power amplifier designs, semi-analytical techniques for wideband microwave power transfer network designs and synthesis with lumped/distributed circuit elements.

Binboga Siddik YARMAN received his B.Sc. in Electrical Engineering from Technical University of Istanbul (1974), M.Sc. degree from Stevens Institute of Technology, NJ, USA (1978), Ph.D. degree from Cornell University, Ithaca, NY, USA (1982). He returned to Turkey in 1984 and served as Assistant, Associate and full Professor at Anatolia University Eskisehir, Middle East Technical
University-Ankara, Technical University of Istanbul, and Istanbul University. He was the Founding President of Isik University. He had been a visiting professor at Ruhr University Bochum, Germany (1987-1994) and Tokyo Institute of Technology, Japan (2006-2008). Currently, he is the chairman of the Department of Electrical-Electronics Engineering of Istanbul University. He holds four US patents assigned to US Air Force. He is the author of the books titled "Design of Ultra Wideband Antenna Matching Networks" by Springer 2008 and "Design of Ultra Wideband Power Networks" by Wiley 2010. He received the Young Turkish Scientist Award in 1986, the Technology Award in 1987 of National Research and Technology Counsel of Turkey. He received the Research Fellowship award of Alexander Von Humboldt Foundation, Germany, in 1987. He became the Member of New York Academy of Science in 1994. He was elevated to IEEE Fellow for his contribution to "Computer Aided Design of Broadband Amplifiers". 